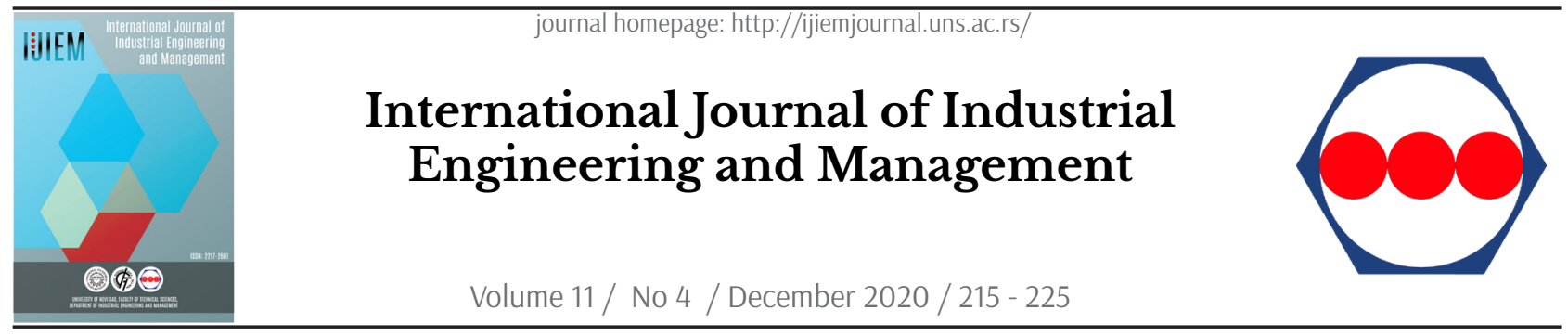

Original research article

\title{
Electric Vehicle Routing Problem with Heterogeneous Vehicles and Partial Charge
}

\author{
H. Park ${ }^{a}$, S. Jin $b *$ \\ a Department of Industrial Engineering, Yonsei University, 50 Yonsei-ro, Seodaemun-gu, Seoul, 03722, S. Korea; \\ b College of Business, Cheongju University, 298, Daeseong-ro, Cheongwon-gu, Cheongju-si, Chungcheongbuk-do, 28503, S. Korea
}

\begin{abstract}
A B STRACT
In this paper, a mathematical formulation is developed to solve an electric vehicle routing problem with heterogeneous vehicles and partial charge to minimize the total distance traveled by the vehicles. The proposed model considers different characteristics of vehicles such as load capacities, battery capacities, energy consumption rates, and charging speeds. In addition, when each vehicle visits the charging station to recharge its battery due to the limitations in the battery capacity, we implement the partial charge, which is the charging method to charge only the necessary amount of energy to finish the remaining route. A numerical analysis is conducted to validate the proposed model and demonstrate its practical applicability.
\end{abstract}

\section{Introduction}

In recent years, researchers have focused on integrating the vehicle routing problem (VRP) and its variants with different environmental aspects to achieve the efficiency, responsiveness, and flexibility [6], [20]. In addition, increase interests towards the management of the internal logistics, which is the connection between the components in the supply chain, have attracted attention to the sustainability [5], [10]. As greenhouse gases and micro dust are large problems, atmospheric pollution has captured global interest. In particular, pollutants emitted from

\section{ARTICLE INFO}

Article history:

Received August 20, 2020

Revised September 30, 2020

Accepted October 2, 2020

Published online October 19, 2020

Keywords:

Electric vehicle routing problem (EVRP);

Heterogeneous vehicles;

Partial charge

*Corresponding author:

Sukho Jin

shjin@cju.ac.kr 
vehicles to electric vehicles because of the possibility of oil depletion and increasing oil prices. The burden of limited resources and the increase in oil prices has increased the interest in energy efficiency, and the energy-efficient electric vehicle market is expanding rapidly.

Many countries in the world have expectations of the environmental and economic effects of electric vehicles and have implemented various policies to increase their usage. As nearly $23 \%$ of the world's carbon emissions are produced by the transportation sector, the increase in electric vehicles in the industry will contribute significantly to reducing greenhouse gas emissions, which will aid in alleviating atmospheric pollution problems and increasing the efficiency of energy usage. Additionally, because of increasing number of goods transported resulting from increasing volume of online delivery, electric vehicles will significantly contribute to reducing costs and environmental pollution in the transportation industry. Currently, a low percentage of transportation vehicles are electric, but with an increasing consensus on the demand for electric vehicle delivery, the penetration rate of replacing transport trucks with electric vehicles in the industry is expected to increase gradually. Figure 1 shows the the grow in number of electric vehicles in the world [4].

The EVRP must consider the characteristics of the electric vehicles such as the limited mileage, limited charging speed, and high initial investment cost. The traveling distance of electric vehicles is limited by their battery power, and the vehicles may need to detour to a charging station to recharge. The charging speed varies depending on the type of vehicle and battery technology. To increase the use of electric vehicles, charging stations must be installed and the following cost must be considered. Therefore, effective operations of transportation networks that reflect the realistic constraints of electric vehicles can sig- nificantly contribute to promoting the use of electric vehicles. In this paper, we propose an EVRP model to minimize the total traveling distance of vehicles considering different loads, battery capacities, energy consumption per distance, and charging speeds depending on the types of vehicles. The model also considers that vehicles can partially charge to the energy required for its remaining travel distance instead of fully charging at the charging station. In the study, we conducted experiments to analyze the factors that can be considered in decision making by logistics operators of electric vehicles.

The remainder of this paper is organized as follows. In Section 2, we present a review of the relevant literature. Section 3 describes the EVRP with a partial charge to minimize the total distance of vehicles. Section 4 presents the numerical experiments and analysis of the results and Section 5 presents the conclusions of the paper and future studies.

\section{Literature review}

This paper addresses the EVRP, which aims to serve a set of customers using electric vehicles. EVRP was introduced recently owing to the commercialization of electric vehicles. The use of electric vehicles is widely increasing in urban freight distribution because the minimization of energy is a key concern for governments [1]. The EVRP is a variant of the well-known VRP, which is a combinatorial problem known to be a generalized version of the traveling salesman problem [12]. It aims to minimize the total operating cost while deciding the set of optimal vehicle routes to deliver products to customers. The VRP has many variants addressing different types of constraints and specifications. One of the variants is the open VRP, in which vehicles are not required to return to the depot. The multi-depot VRP has mul-

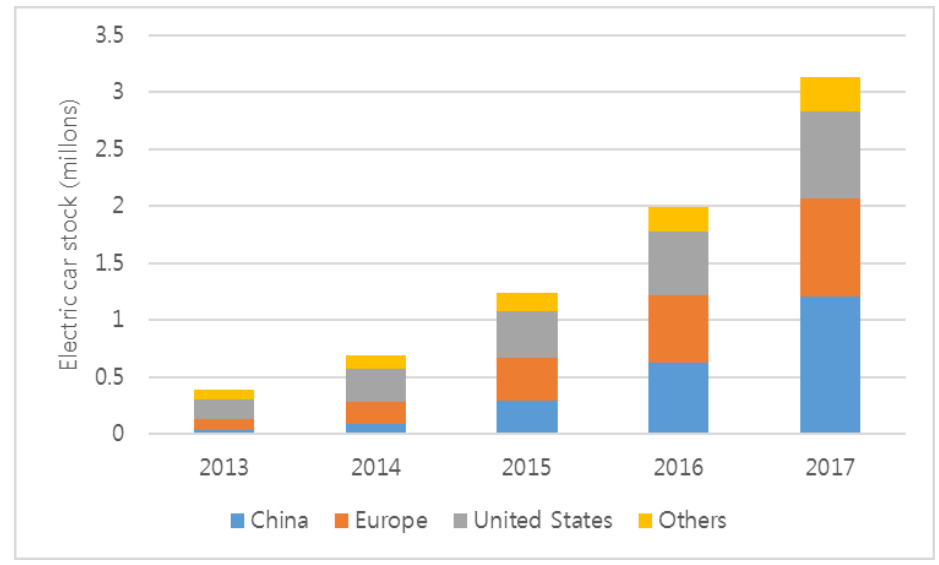

Figure 1. Number of electric vehicles on the road to grow 
tiple depots where vehicles can start and finish the route [21]. The capacitated VRP has a vehicle-weight constraint, and the VRP with heterogeneous vehicles involves operation using vehicles with different characteristics, such as different weight capacities and vehicle speeds [8]. Existing studies related to the proposed model can be divided into types: studies on the EVRP, studies related to VRPs with heterogeneous vehicles, and studies on the EVRP, which enables vehicles to be charged partially at a charging station.

Because of environmental concerns and the recent technological development in the domain of electric vehicles, many researches for the EVRP in a wide variety of domains have been studied [2], [3]. Additionally, many researchers focused their efforts on realistic aspects. Wen et al. [27] proposed a model to derive electric bus schedules with various departure and arrival points for each bus. Shao et al. [25] proposed the VRP with consideration to vehicle travel and charging times, which dynamically change owing to traffic congestion. They proved that the charging method, charging speed, and the number of charging stations were important factors in the operation of electric vehicles by verifying the model and analyzing the results through a case study that reflected an actual traffic scenario. Montoya et al. [19] investigated a model that determined the route to the charging station by considering the driving distance constraints of the electric vehicles. The charging time was considered as a non-linear function, and its importance was demonstrated through comparisons with previous studies. Koc et al. [15] also used the non-linear function for the charging time and considered a shared charging station used by different companies. Lin et al. [17] proposed an EVRP considering the effect of load-carrying capacity on the consumption of electric vehicle batteries. The use of electric vehicles was verified by comparing the maximum travel time and distance according to the load-carrying capacity of trucks powered by diesel and those powered by electricity in actual traffic conditions.

In the transportation industry, vehicles may be divided into different classes according to their load capacity and usage. Reviewing the studies that considered the constraints and scenarios that may occur when heterogeneous vehicles are used [29], Hiermann et al. [10] proposed the vehicle routing model with time constraints for all customers using electric vehicles with different purchasing costs depending on their capacity and battery sizes. Jie et al. [11] developed an optimal delivery strategy for operating heterogeneous vehicles with different battery replacement costs depending on the vehicles' load capacities, battery capacities, and power consumption. Xiao and Konak [28] proposed a pickup and delivery VRP with heterogeneous electric vehicles to minimize greenhouse gas emissions. Variations in traffic congestion over time, time constraints for the vehicles and customers, and load and distance constraints for electric vehicles were considered to create a more realistic environment. Sassi et al. [22] considered conventional gasoline-powered and electric vehicles. They proposed a model considering a varying charging cost over time and a constraint in that the electric charging stations and electric vehicles must be compatible for charging to occur.

Keskin and Catay [13] proposed a model considering partial charging, instead of full charging, of electric vehicles at a charging station, which enabled vehicles to satisfy the customer time constraint. Keskin and Catay [14] further researched the model by considering different charging technologies with different charging speeds. Macrina et al. [18] studied the VRP for a combination of gasoline-powered and electric vehicles. The model facilitated the partial charge of the electric vehicles, and the study compared the environmental value in electric vehicles by comparing two types of vehicles. Schiffer and Walther [23] investigated the EVRP by deciding the optimal routes and the location of the charging station at the same time. They verified that by considering the partial charge of electric vehicles and appropriate locations of the charging station, the model could derive the minimum traveling distance of the vehicles. Felipe et al. [9] studied the model considering several charging methods and partial charge. The model determined the optimal routes and the charging technology at the charging station while minimizing the cost of operating the vehicles to deliver products. Desaulniers et al. [7] considered and varied the frequency of visits to a charging station and the amount of battery charge for electric vehicles to create four scenarios.

The VRP is a problem of determining the route of vehicles while visiting distributed sites to satisfy customer demand, and its general objective is to minimize their traveling distance. Several different studies have been conducted to consider additional constraints, such as the vehicle load capacity, customer time constraint, and heterogeneous vehicles. In particular, several studies on the electric vehicle as the transportation mode have been conducted because constraints on the characteristics of the electric vehicle, such as the limit in travel distance owing to battery capacity, altered routes to visit the charging station, and charging method and speed, must be considered. In addition, the rapid expansion of the 
electric vehicle market and increase in electric vehicle usage has led to further active research. However, based on the aforementioned literature review related to the EVRP considering heterogeneous vehicles and partial charge, a study considering the two aspects simultaneously is necessary to create a more realistic operation environment for electric vehicles. Therefore, this paper proposes an EVRP considering partial charge by relaxing a constraint to charge fully when a vehicle visits a charging station and heterogeneous vehicles with different load capacities, battery capacities, energy consumption per travel distance, and battery charging speeds at a charging station. In addition, the model was verified through numerical experiments, and the effects of the constraints considered were analyzed.

\section{Electric vehicle routing problem}

This study developed an EVRP model to minimize the total traveling distance of electric vehicles while satisfying all customer demands. The proposed model considers the realistic features of electric vehicles. Electric vehicles can detour to charging stations to overcome the traveling distance constraint of the battery capacity, and vehicles can partially charge to the required energy. In addition, heterogeneous vehicles must be used to consider various vehicles existing in the logistics system. These vehicles have different load and battery capacities, battery consumption per traveling distance, and charging speeds. The objective of the proposed EVRP model is to determine the optimal vehicle routes, amount of energy charged to complete the route, and the amounts of products transported while minimizing the total traveling distance of the vehicles.

\subsection{Problem description}

The proposed model uses the distance calculated by using the Euclidean distance formula based on the coordinates of each customer location. Only a single depot exists, and each vehicle must begin at and return to the depot. Each heterogeneous vehicle has a different load capacity, battery capacity, energy consumption, and charging speed. The amount of product transported by a vehicle cannot exceed its load capacity and the amount of energy consumed in the total distance traveled cannot exceed the battery capacity. The number of visits to the charging station is limited to one per vehicle. Each customer can be visited more than once depending on the load and battery capacities, and all demands must be satisfied.
Each vehicle cannot exceed the maximum traveling time limit.

\subsection{Model formulation}

The proposed model is a modification and extension of a model presented in Felipe et al. [9]. Some of the parameters introduced are commonly used in EVRP researches dealing with the partial charge, and constraints presenting these parameters are altered and formulated to fit the model in this study. Energy consumed by a vehicle traveling between nodes and charging stations $\left(\tau_{\mathrm{ijv}}\right.$ and $\left.\tau_{\mathrm{jrv}}^{\mathrm{r}}\right)$ is calculated with the Euclidean distance between the locations and a coefficient (expressed in KWh per $\mathrm{km}$ ) [18]. The amount of energy to charge $\mathrm{y}_{\mathrm{rv}}$ is determined by level of battery remaining when leaving a node $\mathrm{ye}_{\mathrm{jv}}$ and a charging stations $\mathrm{yl}_{\mathrm{rv}}$ and the battery capacity of a vehicle $B_{v}[14]$. Charging speed of a vehicle is used to calculate the time a vehicle is spending at the charging station. Amount of energy charged to finish the remaining route $y_{\mathrm{rv}}$ and charging speed of a vehicle $\rho_{\mathrm{v}}$ compute the charging time at the charging station [2]. Vehicles stays at the charging station only for the amount of energy necessary. Proposed model considers the heterogeneous vehicles (v) and different types of vehicle have different characteristics depending on its size. These characteristics are implemented to the appropriate variables and constraints to fit the model. Intuitively, load and battery capacities are proportional to the vehicle size. Battery consumption rate and charging speeds are also proportional to the vehicle size. We assume the same charging technology but different rate for each vehicle. In addition, charging stations $\mathrm{r}$ are separated from the customer nodes. The behavior of vehicles in different sizes when visiting a charging station alters the vehicle route and affects the results. Changes in energy level and service time for a vehicle at a charging station can be observed in more detail. The following notation is used to formulate the proposed model. The sets, indices, and parameters used to formulate the model are described below.

$$
\begin{aligned}
& \mathrm{i}, \mathrm{j}=\text { index of customers }(\mathrm{i}, \mathrm{j}=0, \ldots, \mathrm{N}) \\
& \mathrm{r}=\text { index of charging stations }(\mathrm{r}=1, \ldots, \mathrm{R}) \\
& \mathrm{v}=\text { index of electric vehicles }(\mathrm{v}=1, \ldots, \mathrm{V}) \\
& \mathrm{q}_{\mathrm{j}}=\text { customer demand at node } \mathrm{j}(\mathrm{kg}) \\
& \mathrm{d}_{\mathrm{ij}}=\text { distance between nodes } \mathrm{i} \text { and } \mathrm{j}(\mathrm{m})
\end{aligned}
$$


$\mathrm{d}_{\mathrm{jr}}=$ distance between node $\mathrm{j}$

and charging station $\mathrm{r}(\mathrm{m})$

$\mathrm{Q}_{\mathrm{v}}=$ capacity of vehicle $\mathrm{v}(\mathrm{kg})$

$B_{v}=$ battery capacity of vehicle $\mathrm{v}(\mathrm{kWh})$

$\mathrm{T}=$ maximum traveling time of vehicles (min)

$\mathrm{tt}_{\mathrm{ij}}=$ traveling time between nodes $\mathrm{i}$ and $\mathrm{j}(\mathrm{min})$

$\operatorname{ttr}_{\mathrm{jr}}=$ traveling time between node $\mathrm{j}$

and charging station $r(\mathrm{~min})$

$\mathrm{s}_{\mathrm{j}}=$ service time at node $\mathrm{j}(\mathrm{min})$

$\rho_{\mathrm{v}}=$ charging speed of vehicle $\mathrm{v}(\mathrm{kWh} / \mathrm{hr})$

$\tau_{\mathrm{ijv}}=$ energy consumption traveling between nodes $\mathrm{i}$ and $\mathrm{j}$ by vehicle $\mathrm{v}(\mathrm{kWh})$

$\tau_{\mathrm{jrv}}^{\mathrm{r}}=$ energy consumption traveling between node $\mathrm{j}$ and charging station $\mathrm{r}$ by vehicle $\mathrm{v}(\mathrm{kWh})$

$\mathbf{M}=$ sufficiently large number
The variables in the model are described below.

$\mathrm{x}_{\mathrm{ijv}}=1$ if vehicle $\mathrm{v}$ is traveling from node $\mathrm{i}$ to node j; 0 otherwise

$\mathrm{u}_{\mathrm{jrv}}=1$ if vehicle $\mathrm{v}$ is traveling from node $\mathrm{j}$ to charging station $\mathrm{r}$; 0 otherwise

$\mathrm{y}_{\mathrm{rv}}=$ amount of energy charged at $\mathrm{r}$ for vehicle $\mathrm{v}$

$\mathrm{ye}_{\mathrm{jv}}=$ amount of energy remaining in vehicle $\mathrm{v}$ when leaving node $\mathrm{j}$

$\mathrm{yl}_{\mathrm{rv}}=$ amount of energy remaining in vehicle $\mathrm{v}$ when leaving charging station $r$

$t_{r v}=$ time when vehicle $v$ leaves charging station $r$

$\mathrm{te}_{\mathrm{jv}}=$ time when vehicle $\mathrm{v}$ leaves node $\mathrm{j}$

$\mathrm{l}_{\mathrm{jv}}=$ amount of product in vehicle $\mathrm{v}$ when leaving node $\mathrm{j}$

$b_{j}=$ auxiliary variable used to eliminate subtours

Based on the notation described, the model for the EVRP with heterogeneous vehicles and partial charge is formulated as follows:

$\operatorname{minimize} \sum_{j} \sum_{r} \sum_{v} x_{i j v} * d_{i j}+\sum_{i} \sum_{j} \sum_{v} u_{j r v} * d_{j r}$

subject to

$$
\begin{gathered}
\sum_{i} \sum_{j} x_{i j v} \geq 1, \quad \forall \mathrm{v} \in \mathrm{V} \\
\sum_{i} x_{i j v}-\sum_{i} x_{j i v}=0, \quad \forall \mathrm{j} \in \mathrm{N}, \mathrm{v} \in \mathrm{V}, \mathrm{i} \neq \mathrm{j} \\
\sum_{j} x_{0 j v}=1, \quad \forall \mathrm{v} \in \mathrm{V} \\
\sum_{i} x_{i 0 v}=1, \quad \forall \mathrm{v} \in \mathrm{V} \\
b_{j} \geq b_{j}+1-M *\left(1-\sum_{v} x_{i i v}\right), \forall \mathrm{i} \in \mathrm{N}, \mathrm{j} \in \mathrm{N} \\
\sum_{j} l_{j v} \geq \sum_{i} l_{i v}+\sum_{j} q_{j}, \quad \forall \mathrm{v} \in \mathrm{V} \\
\sum_{v} l_{j v} \geq q_{j}, \quad \forall \mathrm{j} \in \mathrm{N} \\
\sum_{j} l_{j v} \leq Q_{v}, \quad \forall \mathrm{v} \in \mathrm{V}
\end{gathered}
$$




$$
\begin{aligned}
& l_{j v} \leq M * \sum_{i} x_{i j v}, \quad \forall \mathrm{j} \in \mathrm{N}, \mathrm{v} \in \mathrm{V} \\
& \sum_{j} \sum_{r} u_{j r v} \leq 1, \quad \forall \mathrm{v} \in \mathrm{V} \\
& \sum_{i} \tau_{i j v} * x_{i j v}=y e_{j v}, \quad \forall \mathrm{j} \in \mathrm{N}, \mathrm{v} \in \mathrm{V} \\
& \sum_{j} y e_{j v}-B_{v}=\sum_{r} y_{r v}, \quad \forall \mathrm{v} \in \mathrm{V} \\
& \frac{\mathrm{y}_{r v}}{M} \leq \sum_{j} u_{j r v} \leq M * y_{r v}, \quad \forall \mathrm{r} \in \mathrm{R}, \mathrm{v} \in \mathrm{V} \\
& \mathrm{y}_{\mathrm{rv}} \leq \sum_{j} B_{v} * u_{j r v}, \quad \forall \mathrm{r} \in \mathrm{Rv} \in \mathrm{V} \\
& y_{r v}+\sum_{j} \tau_{j r v}^{r} * u_{j r v}=y l_{r v}, \forall \mathrm{j} \in \mathrm{N}, \mathrm{r} \in \mathrm{Rv} \in \mathrm{V} \\
& \sum_{i}\left(t t_{i j}+s_{j}\right) * x_{i j v}=t e_{j v}, \quad \forall \mathrm{j} \in \mathrm{N}, \mathrm{v} \in \mathrm{V} \\
& \sum_{j} t t r_{j r} * u_{j r v}+\frac{1}{\rho_{v}} * y l_{r v}=t_{r v}, \forall \mathrm{r} \in \mathrm{R}, \mathrm{v} \in \mathrm{V} \\
& 0 \leq t e_{j v} \leq T \forall \mathrm{j} \in \mathrm{N}, \mathrm{v} \in \mathrm{V} \\
& x_{i j v}, u_{j r v}=\{0,1\} \\
& z_{r v}, y_{r v}^{A}, y_{r v}^{L}, y e_{j v}^{A}, y e_{j v}^{L}, t e_{j v}, t_{r v}, l_{j v} \geq 0
\end{aligned}
$$

The objective function (1) seeks to minimize the total traveling distance of vehicles. All the vehicles must travel to nodes to satisfy customer demands and travel to charging stations to recharge their batteries. Constraints (2) to (10) form vehicle routes. Constraint (2) ensures that each customer is visited at least once. More than one vehicle can be used to satisfy the demand according to their load. Constraint (3) guarantees the flow of vehicles, meaning that a vehicle arriving at a node leaves the node. Constraints (4) and (5) ensure that every vehicle begins and ends at the depot. Constraint (6) prevents subtours within the VRP. Constraint (7) and (8) guarantee the amount of demand at each node and the amount transported by vehicles at each node and all customer demands must be satisfied. Constraint (9) guarantees that the overall load capacity of a vehicle along a route is not exceeded. Constraint (10) guarantees that a vehicle has products being transported only if a vehicle trav- els between two corresponding nodes and ensures linearization.

Constraints (11) to (16) relate to the charging of electric vehicles and their visit to the charging stations. Constraint (11) limits the number of visits to charging stations for each vehicle to once. Constraint (12) represents the amount of energy consumed when traveling from one node to another. The energy consumed when traveling from one node to another is calculated using the energy consumption rate per distance unit. Constraint (13) ensures that the difference between the total amount of energy consumed and the battery capacity of a vehicle is equal to the amount of energy charged. This indicates that when the amount of energy required for a vehicle to travel its route is lower than its battery capacity, the amount charged at the charging station is determined by considering the remaining distance to complete the route. Each vehicle has a different battery capacity. Constraint (14) indicates that a visit to a charging station is determined by whether a vehicle requires charging and ensures 
the linearization. Constraint (15) guarantees that the amount of charge at a charging station does not exceed the battery capacity of a vehicle. Constraint (16) indicates the amount of energy consumed when visiting a charging station, and it is added to the total amount of energy consumed by visiting customers. A vehicle visiting charging stations already requires charging owing to a shortage of energy; therefore, the amount of energy consumed to visit charging stations is also replenished through same charging.

Constraints (17) to (19) are the time-related constraints occurring in the operation of the vehicles. Constraint (17) indicates that when a vehicle is traveling to a node, the sum of vehicle travel time and service time is the time the vehicle leaves the node. Constraint (18) represents the time related to when a vehicle visits a charging station. When a vehicle is traveling to a charging station, the sum of vehicle travel time and charging time is the time the vehicle leaves the station. Charging time is calculated using the charging speed $(\mathrm{kWh} / \mathrm{hr})$ and the amount of energy charged. Constraint (19) ensures that a vehicle does not exceed its maximum vehicle time. Constraint (20) represents the binary variables, and constraint (21) indicates the non-negative variables.

Figure 2 shows an example of the proposed model with five customer nodes, two charging stations, a depot, and two electric vehicles. Each vehicle has different battery capacities and detours to a charging station because the energy consumption exceeds the battery capacity. The solid line represents the route a vehicle proceeds as it visits the charging station, and the dotted line represents the original route before the detour. At the charging station, vehicle 1 is partially charged by $8.8 \%$ of its battery capacity vehicle
2 is partially charged by $3.3 \%$ of its battery capacity, including the amount of energy consumed traveling to the charging station. Thereafter, the route is completed by returning to the depot.

\section{Experiment results and discussion}

In this research, we conducted an experimental analysis to verify the mathematical model developed for the EVRP. An optimization software package, ILOG OPL CPLEX Optimization Studio, was used to determine the optimal solution for the developed model.

\subsection{Base experiment}

Experiments were conducted to verify the proposed mathematical model, and its validity was verified by changing the number of customer nodes, charging stations, and electric vehicles. Coordinates of the demand locations and charging stations were randomly generated. The distances between locations (customer nodes, charging stations, a depot) were calculated using the generated coordinates and the Euclidean distance formula. Each customer node had a demand to be satisfied and a service time. As an example of customer node information, five customer nodes, two charging stations, and two electric vehicles for EVRP are shown in Table 1.

The time required by the electric vehicles for traveling between locations was calculated using the travel distance and the average vehicle speed. We assumed that each vehicle was a different type, but they all had the same average traveling speed. Different

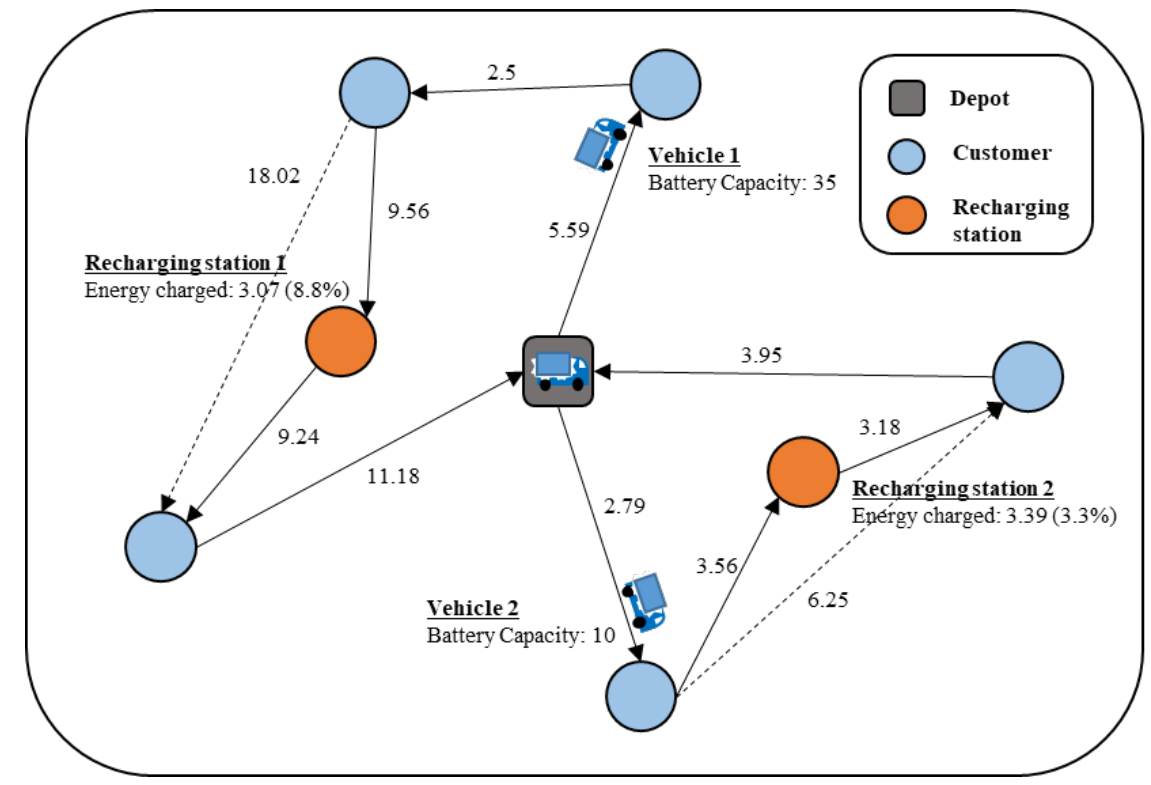

Figure 2. Illustration of EVRP with heterogeneous vehicles and partial charge 
types of electric vehicles had different load capacities, battery capacities, energy consumption per traveling distance, and charging speeds. The vehicles were categorized into small, medium, and large in terms of size, and the characteristics of each vehicle are shown in Table 2. The time limit for each vehicle was considered using the working time of a driver for one day. Data related to the electric vehicles were based on the data obtained from Senn et al. [24] and were altered to fit the problem characteristics of this study.

Table 3 shows the result of the example problem. The result shows routes formed for two vehicles while satisfying all the demands and charging stations visited once by each vehicle. In addition, the proposed model is validated by showing the changes in the amount of energy consumed and travel time when traveling to the next location.

The routes of each vehicle began at the depot; thus, they are omitted from the table. Vehicle 1 visited the customer nodes 1,2 , and 4 and returned to the depot. It detoured to charging station 1 between customer nodes 1 and 2 to partially charge and continued its route. Additionally, the table shows the amount of demand satisfied when a vehicle visited the customer nodes, energy consumed, and vehicle travel time. Vehicle 1 was a small vehicle and the result indicated that the energy consumption was lower than that of vehicle 2 , which was a medium vehicle. Although energy consumption of Vehicle 1 was low, it had to charge at a charging station to complete its route because of a low battery capacity. Additionally, customer node 1 , already visited by vehicle 1 , was visited by vehicle 2 again. Vehicle 1 , with smaller capacity, could satisfy only part of the demand and vehicle 2 visited to satisfy the remainder.

Based on the validated mathematical model, a basic experiment was conducted by changing the number of customer nodes, charging stations, and vehicles to compare the total travel distances of the vehicles and the total energy charged at charging stations. Locations of the customer nodes and charging stations were randomly generated. The experiment was conducted by increasing the number of customer locations, and the viable numbers of charging stations and vehicles were determined according to the number of customer locations. Table 4 shows the experi-

Table 1. Demand information for 5 customers, 2 charging stations, and 2 vehicles for the EVRP

\begin{tabular}{cccc}
\hline Customer & $\begin{array}{c}\text { Location } \\
(\mathrm{x}, \mathrm{y})\end{array}$ & Demand $(\mathrm{kg})$ & Service time (min) \\
\hline Depot & $(45,40)$ & 0 & 0 \\
1 & $(10,78)$ & 700 & 10 \\
2 & $(8,18)$ & 600 & 10 \\
3 & $(56,60)$ & 500 & 10 \\
4 & $(34,70)$ & 800 & 10 \\
\hline 5 & $(58,38)$ & 900 & - \\
\hline
\end{tabular}

Table 2. Characteristics of electric vehicles (trucks) in different sizes: small, medium, and large

\begin{tabular}{cccc}
\hline & Small & Medium & Large \\
\hline Average Speed $(\mathrm{km} / \mathrm{h})$ & 25 & 0 & 0 \\
Vehicle Capacity $(\mathrm{kg})$ & 1000 & 5000 & 10000 \\
Battery Capacity $(\mathrm{kWh})$ & 60 & 250 & 450 \\
Energy consumption $(\mathrm{kWh} / \mathrm{km})$ & 0.33 & 0.83 & 1.00 \\
Recharging rate $(\mathrm{kWh} / \mathrm{h})$ & 3.6 & 20 & 45 \\
Time limit $(\mathrm{m})$ & 600 & 900 & 10 \\
\hline
\end{tabular}


ment results. $\mathrm{N}$ represents the customer nodes, $\mathrm{R}$ the charging stations, and $\mathrm{K}$ the vehicles. The total travel distance and amount of energy charged proportionally increased as the number of customer nodes increased. The results indicated that the total distance traveled when the number of customer nodes was 8 was lower than for 6 or 7 customer nodes. The distance to the charging station was farther and the amount of energy charged increased proportionally to the distance traveled. The scenario with six customer nodes had fewer charging stations and the total distance traveled and the amount of energy charged increased because the charging station was farther. For the scenario with five customer nodes, a large difference in the total distance traveled was observed. A smaller number of vehicles reduced the distance traveled to and from the depot. The larger total distance traveled for all examples resulted in a larger total energy charged, but more customer nodes did not necessarily have larger total distance traveled. In addition, more vehicles did not necessarily have less total energy charged. Through the base experiment, the proposed model was validated with the changes in the number of customer nodes. Number of charging stations and vehicles had a significant effect on the amount of energy charged at the charging stations depending on the distance between the locations and the number of vehicles.

\subsection{Analysis of vehicle type combinations}

We analyzed the effect of the change in the combination of the vehicle types on the total distance traveled and the amount of energy charged at charging stations. The N6R2K3 example was used to analyze the combination of small, medium, and large vehicles.

As Table 5 shows, six combinations were created for the experiment. The results indicated that the combination used in the basic experiment had the longest distance traveled and the second most amount of energy charged. In the scenarios operating with three large vehicles, the solution was infeasible as slow charging time made completing the route within the maximum time limit of a vehicle impossible. As the number of small vehicles increased, the total distance traveled and the amount of energy charged decreased. However, comparing the scenario of three small vehicles and that of two small vehicles and one medium vehicle, the amount of energy charged

Table 3. Optimal vehicle routes for 5 customers, 2 charging stations, and 2 vehicles (small and medium)

\begin{tabular}{|c|c|c|c|c|c|c|c|}
\hline \multicolumn{8}{|l|}{ Vehicle 1 (Small) } \\
\hline Route & 1 & - & 2 & 4 & 0 & - & - \\
\hline Charging Station & - & 1 & - & - & - & - & - \\
\hline Demand satisfied & 100 & - & 400 & 500 & - & - & - \\
\hline Battery consumed & 15.97 & 15.4 & 18.3 & 19.2 & 10.1 & - & - \\
\hline Travel time & 1.94 & 1.87 & 1.19 & 2.33 & 1.22 & - & - \\
\hline \multicolumn{8}{|l|}{ Vehicle 2 (Medium) } \\
\hline Route & 1 & 3 & - & 2 & 5 & 4 & 0 \\
\hline Charging Station & - & - & 2 & - & - & - & - \\
\hline Demand satisfied & 600 & 500 & - & 200 & 900 & 300 & - \\
\hline Battery consumed & 42.6 & 43.5 & 32.9 & 30.1 & 47.4 & 35.2 & 26 \\
\hline Travel time & 1.94 & 1.98 & 1.49 & 1.37 & 2.15 & 1.6 & 1.2 \\
\hline
\end{tabular}

Table 4. Comparison of total distance traveled and total energy charged for the change in the number of customers

\begin{tabular}{lcc}
\hline & Total Distance Traveled $(\mathrm{m})$ & Total Energy Charged $(\mathrm{kWh})$ \\
\hline N5R2K2 & 500.19 & 10.27 \\
N6R2K3 & 982.50 & 35.64 \\
N7R3K3 & 934.24 & 11.27 \\
N8R3K3 & 926.71 & 9.58 \\
\hline
\end{tabular}


for the former was larger because of lower vehicle battery capacities, resulting in visits to the charging stations. The result showed that a combination of vehicle types should be selected considering the vehicle characteristics, travel distance, and energy charged.

\subsection{Analysis of charging method}

We analyzed the effects of partial charging at the charging station. This research considered partial charging that only charges the energy necessary to complete the remaining route.

Table 6 shows the result of an example problem with five customer nodes and two charging stations to compare partial and full charge. Vehicle 1 was small and it charged to $2.80 \mathrm{kWh}$ for the partial charge and $57.20 \mathrm{kWh}$ for the full charge. The corresponding times spent at the charging station were 0.78 and 15.89 hours, respectively. Vehicle 2 was a medium vehicle; thus, its energy consumption per distance traveled, battery capacity, and the difference between the partial and full charge was larger. The amount of energy charged for the partial charging was approximately $4.9 \%$ and $3.7 \%$ of the full charge, respectively. This experiment indicated that the charging method does not affect the total distance traveled, but the partial charging reduces the amount of energy charged and the charging time, which have a significantly positive effect on the total cost. Figure 3 shows the experimental results of the difference between partial and full charges.

\section{Conclusion}

The recent interest in air pollution caused by micro dust and greenhouse gases has drawn keen interest in eco-friendly electric vehicles. Owing to limited resources and continually increasing oil prices, interest in energy efficiency in the transportation industry is also increasing, and the electric vehicle market is expanding rapidly as conventional vehicles are replaced with electric vehicles. However, electric vehicles have limitations in terms of battery capacity and technology, and research on electric vehicles are required to implement solutions in real-life environments. Therefore, in this paper, we propose an EVRP model with heterogeneous vehicles and partial charge. The proposed model is a realistic logistics operation plan model of electric vehicles considering partial charge, which reduces the amount of energy charged at the charging station. Thus, this reduces the charging time. The proposed model was verified through numerical experiments, and the comparative experiments were conducted considering different combinations of vehicle types and charging methods to compare the effects on the decision-making process of logistics managers.

A future study of this research is to develop an algorithm to solve larger instances. As the VRP is a NP-hard problem, one of the meta-heuristic methods that can be used to solve the VRP adequately, such as genetic algorithm or tabu search, can be implement-

Table 5. Comparison of total distance traveled and total energy charged for different combinations of electric vehicles

\begin{tabular}{ccc|cc}
\hline & Vehicle Combination & Total Distance & $\begin{array}{c}\text { Total Energy } \\
\text { Traveled }(\mathrm{m})\end{array}$ \\
\cline { 1 - 2 } Small & Medium & Large & 982.50 & 35.64 \\
1 & 1 & 1 & 834.78 & 32.09 \\
2 & 2 & 0 & 698.76 & 10.64 \\
3 & 1 & 0 & 596.45 & 15.96 \\
0 & 0 & 0 & 974.33 & 40.15 \\
0 & 3 & 0 & - & - \\
\hline
\end{tabular}

Table 6. Comparison of energy charged and recharging time per vehicle for charging methods: partial and full charge

\begin{tabular}{lcccc}
\hline & & Partial Charge & Full Charge & $\%$ \\
\hline \multirow{2}{*}{ Vehicle 1 (Small) } & Energy Charged (kWh) & 2.80 & 57.20 & 4.89 \\
& Recharging Time (hr) & 0.78 & 15.89 & 4.91 \\
\hline \multirow{2}{*}{ Vehicle 2 (Medium) } & Energy Charged (kWh) & 7.47 & 242.53 & 3.08 \\
& Recharging Time (hr) & 0.37 & 12.13 & 3.05 \\
\hline
\end{tabular}


ed. In addition, considering the current increase in the use of electric vehicles, a VRP addressing both conventional combustion-engine and electric vehicles will be a more realistic research and will be more effective in applying real-life data. Also, other realistic factors affecting the efficiency of an electric vehicle battery such as the temperature, humidity, and user's behavior could be considered for an extension of the proposed model.

\section{Funding}

This work was supported by Basic Science Research Program through the National Research Foundation of Korea (NRF) funded by the Ministry of Science and ICT (NRF-2019R1G1A1002234).

\section{References}

[1] Afroditi, Anagnostopoulou, et al. "Electric vehicle routing problem with industry constraints: trends and insights for future research." Transportation Research Procedia 3 (2014): 452-459.

[2] Bruglieri, Maurizio, et al. "A matheuristic for the electric vehicle routing problem with time windows." arXiv preprint arXiv:1506.00211 (2015).

[3] Bruglieri, Maurizio, et al. "A Variable Neighborhood Search Branching for the Electric Vehicle Routing Problem with Time Windows.” Electron. Notes Discret. Math. 47 (2015): 221-228.

[4] Bunsen, Till, et al. "Global EV Outlook 2018: Towards cross-modal electrification.” (2018).

[5] Cano, Jose Alejandro, Alexander Alberto Correa-Espinal, and Rodrigo Andrés Gómez-Montoya. "An evaluation of picking routing policies to improve warehouse efficiency." Int. J. Ind. Eng. Manag 8.4 (2017): 229-238.

[6] Demir, Emrah, Tolga Bektal, and Gilbert Laporte. "A review of recent research on green road freight transportation.” European Journal of Operational Research 237.3 (2014): 775-793.

[7] Desaulniers, Guy, et al. "Exact algorithms for electric vehicle-routing problems with time windows." Operations Research 64.6 (2016): 1388-1405.

[8] Ding, Nan, Rajan Batta, and Changhyun Kwon. "Conflict-free electric vehicle routing problem with capacitated charging stations and partial recharge." Technical Report. Department of Industrial and Systems Engineering, University at Buffalo, US and Department of Industrial and Management Systems Engineering, University of South Florida, US, 2015.

[9] Felipe, Ángel, et al. "A heuristic approach for the green vehicle routing problem with multiple technologies and partial recharges.” Transportation Research Part E: Logistics and Transportation Review 71 (2014): 111-128.

[10] Hiermann, Gerhard, et al. "The electric fleet size and mix vehicle routing problem with time windows and recharging stations." European Journal of Operational Research 252.3 (2016): 995-1018.

[11] Jie, Wanchen, et al. "The two-echelon capacitated electric vehicle routing problem with battery swapping stations: Formulation and efficient methodology.” European Journal of Operational Research 272.3 (2019): 879-904.
[12] Júnior, Alvaro Neuenfeldt, and Lucas Rebouças Guimarães. "A greedy randomized adaptive search procedure application to solve the travelling salesman problem.” Int. J. Ind. Eng. Manag. 10 (2019).

[13] Keskin, Merve, and Bülent Çatay. "Partial recharge strategies for the electric vehicle routing problem with time windows.” Transportation Research Part C: Emerging Technologies 65 (2016): 111-127.

[14] Keskin, Merve, and Bülent Çatay. "A matheuristic method for the electric vehicle routing problem with time windows and fast chargers." Computers \& Operations Research 100 (2018): 172-188.

[15] Koç, Çanrı, et al. "The electric vehicle routing problem with shared charging stations.” International Transactions in Operational Research 26.4 (2019): 1211-1243.

[16] Lin, Canhong, et al. "Survey of green vehicle routing problem: past and future trends." Expert systems with applications 41.4 (2014): 1118-1138.

[17] Lin, Jane, Wei Zhou, and Ouri Wolfson. "Electric vehicle routing problem.” Transportation Research Procedia 12. Supplement C (2016): 508-521.

[18] Macrina, Giusy, et al. "The green mixed fleet vehicle routing problem with partial battery recharging and time windows." Computers \& Operations Research 101 (2019): 183-199.

[19] Montoya, Alejandro, et al. "The electric vehicle routing problem with nonlinear charging function." Transportation Research Part B: Methodological 103 (2017): 87-110.

[20] Morosini, Enzo. "Simulation-Based Analysis of Integrated Production and Transport Scheduling." Int. J. Ind. Eng. Manag 4.3 (2013): 109-116.

[21] Santa Chávez, Jhon Jairo, et al. "A metaheuristic ACO to solve the multi-depot vehicle routing problem with backhauls.” Int. J. Ind. Eng. Manag 6.2 (2015): 49-58.

[22] Sassi, Ons, Wahiba Ramdane Cherif-Khettaf, and Ammar Oulamara. "Iterated tabu search for the mix fleet vehicle routing problem with heterogenous electric vehicles." Modelling, Computation and Optimization in Information Systems and Management Sciences. Springer, Cham, 2015. 57-68.

[23] Schiffer, Maximilian, and Grit Walther. "The electric location routing problem with time windows and partial recharging." European Journal of Operational Research 260.3 (2017): 995-1013.

[24] Senn, L. A. N. F. R. A. N. C. O., et al. "Sviluppare la mobilità elettrica: tecnologie, ambiente, infrastrutture, mercato e regole." (2011).

[25] Shao, Sai, et al. "Electric vehicle routing problem with charging time and variable travel time." Mathematical Problems in Engineering 2017 (2017).

[26] Torres, Diamantino, Ana Raquel Xambre, and Leonor Teixeira. "Development of synchronized logistics scenarios.” Int. J. Ind. Eng. Manag 7.2 (2016): 85-93.

[27] Wen, Min, et al. "An adaptive large neighborhood search heuristic for the electric vehicle scheduling problem." Computers \& Operations Research 76 (2016): 73-83.

[28] Xiao, Yiyong, and Abdullah Konak. "The heterogeneous green vehicle routing and scheduling problem with timevarying traffic congestion.” Transportation Research Part E: Logistics and Transportation Review 88 (2016): 146-166.

[29] Zuo, Xiaorong, et al. "Using AMPL/CPLEX to model and solve the electric vehicle routing problem (EVRP) with heterogeneous ed fleet.” 2017 29th Chinese Control And Decision Conference (CCDC). IEEE, 2017. 